Schlieblich sei hier noch bemerkt, daß in der vorigjährigen Übersicht ${ }^{1}$ ) der Schwankungstahlen bei Kirschsaft unter No. 1 der niedrigste Gehalt an Asche nicht 0,30 sondern $0,35 \mathrm{~g}$ betrug.

A. Bòmer.

1) Diese Zeitschrift 1908, 15, 130.

\title{
Beitrag zur Fruchtsaft-Statistik des Jahrganges 1908.
} Von

\author{
A. Behre, Fr. Große und G. Sclimidt.
}

Mitteilung a as dem Chemischen Untersachungamte der Stadt Chemnitz

Die Untersuchung selbstgepreßter Fruchtsäfte konnte sich in diesem Jahre nur auf Himbeersäfte, Heidelbeersäfte und Preißelbeersäfte erstrecken. Für die Praxis kommen zumeist nur die Himbeersäfte in Frage und es wurde daher von dieser Fruchtart eine größere Anzahl Säfte gepreßt und untersucht als in Vorjahre. Die Himbeeren stammten sämtlich aus der näberen oder weiteren Umgebung von Chemuitz und die aus ihnen gewonnenen vergorenen Säfte können daher mit Recht zur Beurteilung der diesjährigen Ernte, soweit unsere Gegend in Frage kommt, herangezogen werden. Die gepreßten Heidelbeer- und Preißelbeersäfte waren bestimmt, anderweitigen Untersuchungen zu dienen, die aber bisher nicht zum Abschluß gelangt sind. Soweit gleichzeitig die Gesamtanalysen dieser Fruchtsäfte ausgeführt worden sind, finden sie sich in den Tabellen II und III S. 736-737 zusammengestellt.

Bezüglich der Herstellung der Säfte und der bei der Untersuchung angewendeten Verfahren verweisen wir auf die Angaben in unserem vorjährigen Beitrag zur Fruchtsaftstatistik ${ }^{1}$ ). Auch bei den diesjährigen Himbeer- und Heidelbeersäften ist neben der bisher üblichen Methode der Alkalitätsbestimmung das Verfahren von Farnsteiner angewendet worden. Von der Berechnung der Durchschnittszahlen für spezifisches Gewicht, Extrakt und Säure wurde aus den bereits in unserer vorjährigen Veröfentlichung angeführten Gründen -abgesehen. Als die für die vergleichende Beurteilung wesentlichen Werte kommen nur die für Gesamtasche, Aschenalkalität und Alkalitätszahl gefundenen Werte bezw. deren Durchschnittswerte in Betracht. Diese sind daher zusammen mit den Untersuchungsergebnissen der Jahrgänge 1906 und 1907 in der Tabelle IV (S. 735) zusammengefaßt worden.

Die Vergärung der Himbeer- und Heidelbeersäfte ging in dem kühlen, regnerischen August dieses Jahres verhältnismäßig langsam vôn statten. Möglicherweise infolge der dadurch beđingten längeren Vergärungsdauer zeigten verschiedene Säfte große Neigung, essigstichig zu werden, wie aus dem unverhältnismäßig hohen Gehalt einiger Säfte an flüchtiger Säure zu entnehmen ist. Die durch die Titration der Gesamtsäure gefundenen hohen Zahlen dürften demnach erst nach Abzug der flüchtigen Säure (berechnet als Essigsäure) passende Vergleichswerte geben. Eine solche Um- 
rechnung hat demnach bei fast allen Säften stattgefunden und zur Aufnahme einer besonderen Kolumne in den Tabellen geführt.

Die Essigsäure wurde in $50 \mathrm{ccm}$ Saft nach der Vorschrift für Wein bestimmt, nur bei den in der Tabelle II näher bezeichneten Heidelbeersäften wurde der Essigsäureghalt des alkoholischen Destillates ermittelt. Nach unseren Untersuchungen pflegt sich im alkoholischen Destillat aber nur etwa die Hälfte der flüchtigen Säuren vorzufinden, weshalb die Destillation mit Wasserdampf bis auf ein Volumen von $200 \mathrm{ccm}$ Destillat ausgeführt werden muß.

Die Analysenergebnisse sind in den Tabellen I-III auf S. 736-737 wiedergegeben :

Tabelle IV.

\begin{tabular}{|c|c|c|c|c|c|c|c|c|c|c|c|c|}
\hline \multirow[b]{2}{*}{ Bezeichnung der Säfte } & \multicolumn{4}{|c|}{ Jahrgang 1906} & \multicolumn{4}{|c|}{ Jahrgang 1907} & \multicolumn{4}{|c|}{ Jabrgang 1908} \\
\hline & 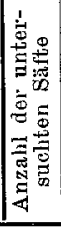 & 总 & 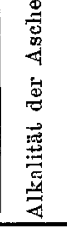 & 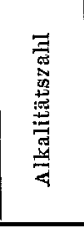 & 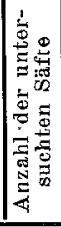 & 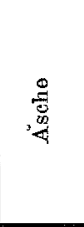 & 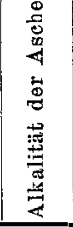 & 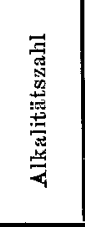 & 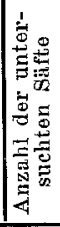 & 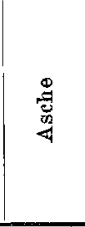 & 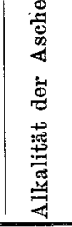 & 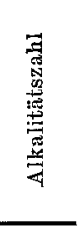 \\
\hline Himbeersäfte & 7 & 0,552 & 6,91 & 12,60 & 10 & 0,546 & 7,26 & 13,30 & 20 & 0,582 & 6,71 & 12,59 \\
\hline Heidelbeersäfte. & 6 & 0,294 & 3,21 & 10,80 & 6 & 0,275 & 3,38 & 12,26 & 10 & 0,286 & 3,37 & 11,75 \\
\hline Preißelbeersäfte , . . & - & - & - & - & 6 . & 0,347 & 2,27 & 6,7 & 6 & 0,276 & 3,88 & 13,69 \\
\hline
\end{tabular}

Die Zusammensetzung der vergorenen $\mathbf{H i m b e e r s a ̈ f t e ~ i s t ~ i n ~ d e n ~ d r e i ~ J a h r - ~}$ gängen hinsichtlich ibres Gehaltes an Asche und Alkalität der Asche keinen großen Schwankungen ausgesetzt gewesen. Die Aschenalkalität des letzten Jahrganges war etwas geringer als die der Vorjahre.

Die Zahlen der Heidelbeersäfte zeigen bei allen drei Jahrgängen nur sehr geringe Unterschiede. Die diesjährigen Preißelbeersäfte jedoch weisen wesentlich geringere Aschengehalte als jim Vorjahre und daneben bedeutend höhere Aschenalkalitäten auf. Infolgedessen ist auch die Alkalitätszabl, welche ja eine außerordentlich empfindliche Verhältniszahl darstellt, viel höher als bei den Säften des Jahrganges 1907 und beträgt etwa das Doppelte der vorjährigen Zahlen. Die Ursache dieser Erscheinung dürfte vielleicht darin $z u$ finden sein, daß in diesem Jahre unvergorene Säfte vorlagen, welche kurze Zeit nach der Pressung untersucht worden waren. Die Preißelbeersäfte vergären bekanntlich wegen ihres Gehaltes an Benzoesäure sehr schwer, bei den diesjährigen gepreßten Säften dieser Fruchtart konnte eine Gärung erst nach etwa vier Wochen beobachtet und nur bei fortgesetzter Luftzufuhr unterbalten werden. Die Untersuchung der Preißelbeersäfte wird sich nach beendeter Gärung nochmals auf Gehalt an Asche und Aschenalkalität erstrecken. Die vorjährigen Preißelbeersäfte zeigten Gehalte an Benzoesäure, die zwischen 0,045 und $0,112 \mathrm{~g}$ in $100 \mathrm{ccm}$ schwankten. 
Tabelle I.

(Hauptgärnng 3-7 Tage;

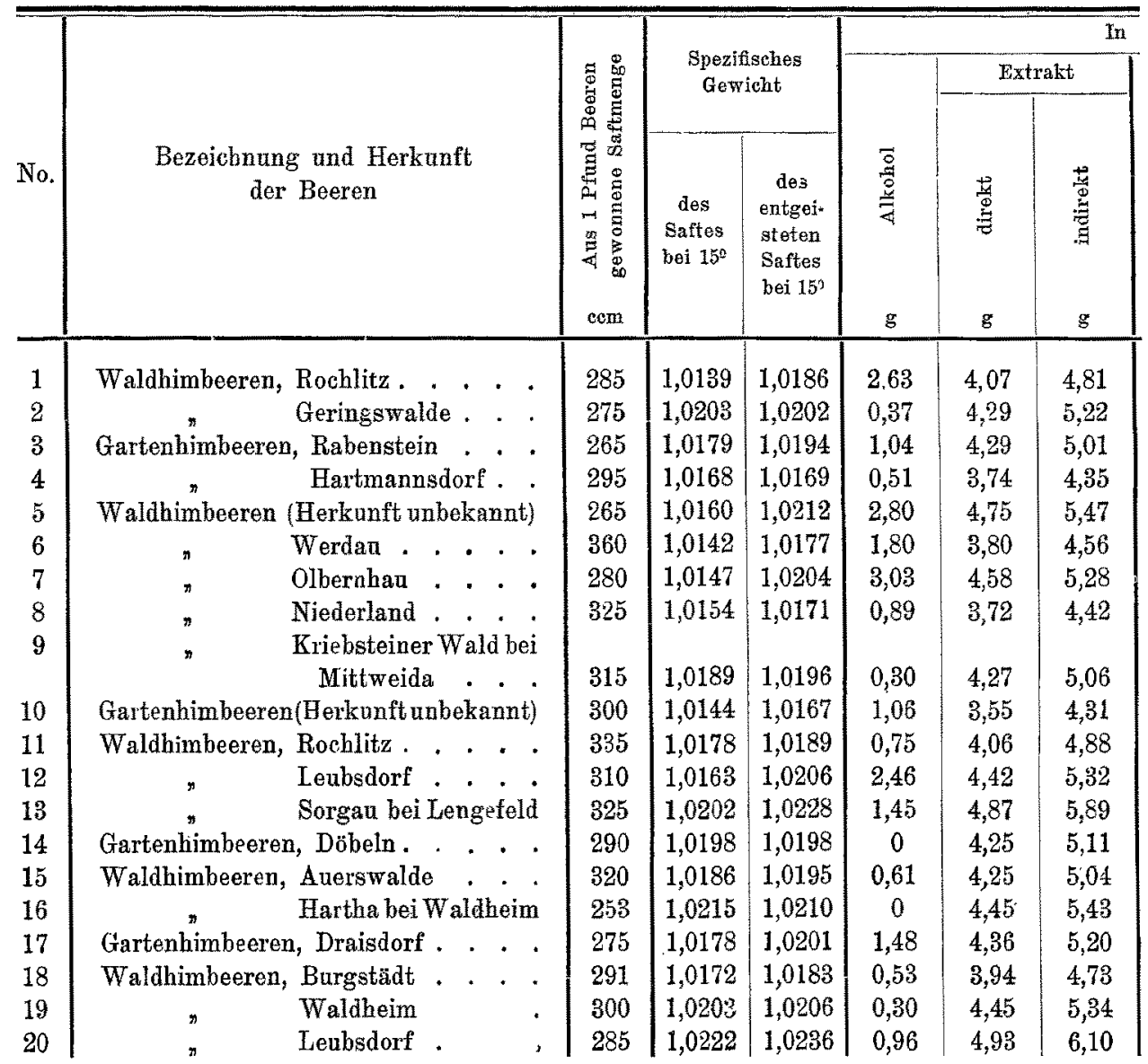

Tabelle II.

(Hauptgärung 8-11 Tage;

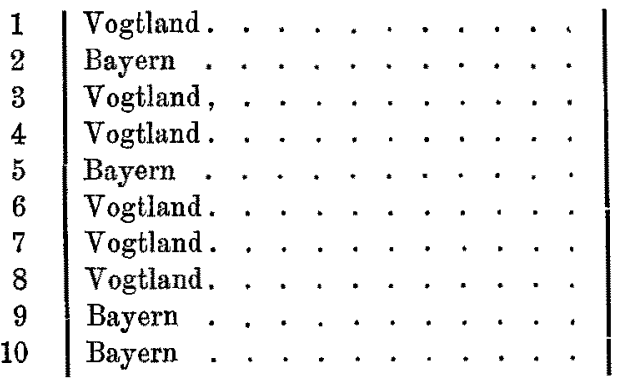

\begin{tabular}{l|l|l|l}
360 & 1,0109 & 1,0156 & 2,72 \\
355 & 1,0151 & 1,0189 & 2,21 \\
385 & 1,0089 & 1,0144 & 3,17 \\
350 & 1,0085 & 1,0141 & 3,23 \\
360 & 1,0129 & 1,0167 & 2,10 \\
370 & 1,0114 & 1,0160 & 2,49 \\
348 & 1,0134 & 1,0188 & 2,97 \\
310 & 1,0158 & 1,0188 & 1,60 \\
310 & 1,0142 & 1,0184 & 2,27 \\
298 & 1,0127 & 1,0185 & 3,17
\end{tabular}

3,77

4,03

4,44

4,88

3,44

3,72

3,37

3,64

$3,84 \quad 4,31$

$3,60 \quad 4,12$

$4,17 \quad 4,86$

$4,17 \quad 4,85$

$4,07 \quad 4,74$

\begin{tabular}{l|l|}
4,33 & 4,77
\end{tabular}

Tabelle IIT.

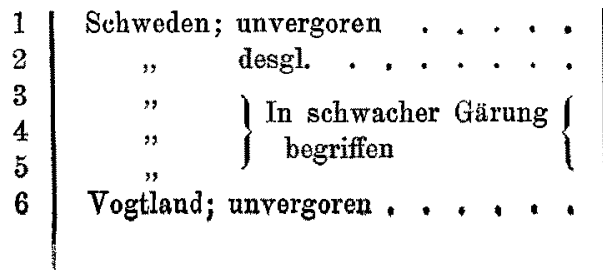

\begin{tabular}{|c|c|c|c|c|c|}
315 & 1,0492 & - & 0 & 12,11 & 12,74 \\
375 & 1,0499 & - & 0 & 12,14 & 12,91 \\
335 & - & - & - & 10,65 & - \\
350 & - & - & - & 12,17 & - \\
375 & - & - & - & 10,51 & - \\
355 & 1,0421 & - & 0 & 9,94 & 10,90
\end{tabular}


Himbeersäfte.

Nachgärung 2-6 Tage.)

\begin{tabular}{|c|c|c|c|c|c|c|c|c|c|c|}
\hline \multicolumn{10}{|c|}{$100 \mathrm{ecm} \mathrm{Saft}$} & \multirow[b]{3}{*}{\begin{tabular}{|c} 
Polarisation \\
naeh der \\
Inversion \\
im $200 \mathrm{~mm}-$ \\
Rohr
\end{tabular}} \\
\hline \multicolumn{2}{|c|}{ Freie Säure } & \multirow[b]{2}{*}{ 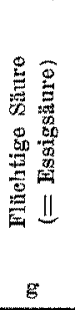 } & \multirow[b]{2}{*}{ 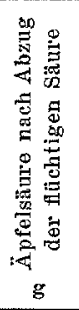 } & \multirow[b]{2}{*}{ 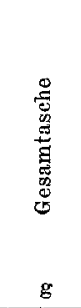 } & \multicolumn{2}{|c|}{$\begin{array}{l}\text { Alkalität der Asche } \\
\text { (ccm N.-Lange) }\end{array}$} & \multicolumn{2}{|c|}{ Allialitätszahl } & \multirow[b]{2}{*}{ 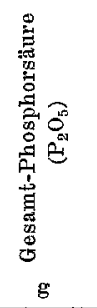 } & \\
\hline 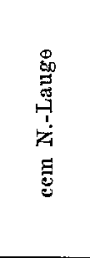 & 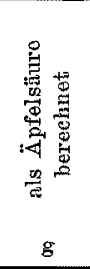 & & & & 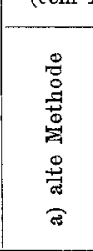 & 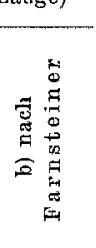 & a & b & & \\
\hline 19,50 & 1,306 & 0,204 & 1,078 & 0,601 & 6,84 & 6,08 & 11,37 & 10,11 & 0,0379 & \pm 0 \\
\hline 43,80 & 2,985 & 1,782 & 0,945 & 0,550 & 6,86 & 5,93 & 12,49 & 11,99 & 0,0171 & $-0,3$ \\
\hline 43,38 & 2,908 & 2,034 & 0,637 & 0,490 & 6,69 & 5,46 & 13,68 & 11,16 & 0,0399 & $-0,25$ \\
\hline 30,50 & 2,044 & 1,149 & 0,761 & 0,431 & 6,00 & 4,90 & 13,93 & 11,38 & - & $-0,2$ \\
\hline 25,75 & 1,726 & 0,129 & 1,582 & 0,580 & 7,40 & 6,94 & 12,75 & 12,25 & 0,0239 & $-0,2$ \\
\hline 20,75 & 1,391 & 0,162 & 1,210 & 0,513 & 6,00 & 5,74 & 11,69 & 11,25 & 0,0176 & \pm 0 \\
\hline 22,25 & 1,492 & 0,342 & 1,110 & 0,579 & 7,40 & 6,98 & 12,79 & 12,13 & 0,0242 & $-0,2$ \\
\hline 29,00 & 1,944 & 0,654 & 1,214 & 0,483 & 5,52 & 5,28 & 11,44 & 11,35 & 0,0202 & $-0,2$ \\
\hline 31,40 & 2,104 & 0,549 & 1,491 & 0,552 & 7,07 & 6,9 & 12,11 & 12,47 & 0,0095 & \pm 0 \\
\hline 25,70 & 1,723 & 0,417 & 1,257 & 0,429 & 5,55 & 5,16 & 12,94 & 11,61 & 0,0255 & $-0,2$ \\
\hline 27,68 & 1,856 & 0,720 & 1,052 & 0,567 & 7,20 & 6,58 & 12,62 & 11,43 & 0,0038 & \pm 0 \\
\hline 28,50 & 1,901 & 0,134 & 1,751 & 0,535 & 6,55 & 6,28 & 11,25 & 11,01 & 0,0321 & $-0,2$ \\
\hline 49,85 & 3,341 & 1,002 & 2,223 & 0,608 & 7,45 & 6,88 & 12,26 & 11,53 & 0,0392 & \pm 0 \\
\hline 85,40 & 2,373 & 0,366 & 1,964 & 0.531 & 7,03 & 6,62 & 13,24 & 12,61 & 0,0112 & $-0,4^{0}$ \\
\hline 41,82 & 2,803 & 0,843 & 1,862 & 0,509 & 6,03 & 6,00 & 11,85 & 11,84 & 0,0125 & \pm 0 \\
\hline 35,40 & 2,373 & 0,629 & 1,671 & 0,635 & 8,24 & 8,20 & 13,02 & 12,87 & 0,0214 & $-0,4$ \\
\hline 45,80 & 3,070 & 0,850 & 2,121 & 0,461 & 5,49 & 5,36 & 11,87 & 11,44 & 0,0221 & $-0,1$ \\
\hline 32,82 & 2,602 & 0,597 & 1,935 & 0,463 & 6,09 & 5,70 & 13,14 & 12,12 & 0,0137 & $-0,2$ \\
\hline 34,10 & 2,286 & 0,472 & 1,759 & 0,543 & 7,34 & 7,16 & 13,51 & 13,02 & 0,0101 & $-0,2$ \\
\hline 55,82 & 3,741 & 1,483 & 2,046 & 0,625 & 7,39 & 6,36 & 11,81 & 10,11 & 0,0486 & \pm 0 \\
\hline
\end{tabular}

Heidelbeersäfte.

Nachgärung 6-10 Tage.)

\begin{tabular}{|l|l|l|l|l|l|l|l|r|r|r}
20,00 & $\mathbf{1 , 3 4 0}$ & 0,270 & 1,039 & 0,290 & 3,62 & 3,16 & 12,31 & 10,90 & -- & $-0,150$ \\
21,88 & 1,467 & 0,354 & 1,072 & 0,343 & 3,74 & 3,40 & 10,40 & 9,91 & - & $-0,300$ \\
16,83 & 1,128 & 0,050 & 1,068 & 0,264 & 3,08 & 2,89 & 11,67 & 10,95 & - & $-0,050$ \\
17,63 & 1,182 & 0,078 & 1,095 & 0,264 & 2,95 & 2,58 & 11,17 & 9,77 & - & $-0,200$ \\
28,70 & 1,924 & 0,819 & 1,009 & 0,281 & 3,24 & 2,82 & 11,58 & 10,04 & - & $-0,200$ \\
19,00 & 1,273 & $\left.0,030^{1}\right)$ & - & 0,285 & 3,17 & 2,75 & 11,12 & 9,65 & - & $-0,750$ \\
20,50 & 1,374 & $0.1021)$ & - & 0,275 & 3,37 & 3,08 & 12,24 & 11,20 & - & $-0,550$ \\
28,00 & 1,876 & 1,035 & 0,841 & 0,281 & $\mathbf{3 , 3 3}$ & 3,16 & 11,50 & 11,23 & - & $-0,250$ \\
25,25 & $\mathbf{1} 692$ & 0,441 & 1,251 & 0,281 & 3,65 & 3,31 & 13,00 & 11,78 & - & $-0,900$ \\
28,25 & $\mathbf{1 , 8 9 3}$ & $\left.0,102^{1}\right)$ & - & 0,295 & 3,55 & 3,34 & 12,03 & 11,32 & - & $-0,700$
\end{tabular}

Preißelbeersäfte.

\begin{tabular}{|l|l|l|l|l|l|l|l|l|l|l|l}
32,50 & 2,275 & $0,071^{1}$ & - & 0,307 & 4,35 & - & 14,17 & - & - & - \\
28,25 & 1,893 & $\left.0,056^{1}\right)$ & - & 0,260 & 3,60 & - & 13,84 & - & - & - \\
28,50 & 1,910 & - & - & 0,255 & 3,45 & - & 18,10 & - & - & - \\
33,25 & 2,228 & - & - & 0,297 & 3,90 & - & 13,10 & - & - & - \\
33,00 & 2,211 & - & - & 0,250 & 3,60 & - & 14,40 & - & - & - \\
32,50 & 2,275 & $\left.0,018^{4}\right)$ & - & 0,325 & 4,40 & - & 13,54 & - & - & -
\end{tabular}

1) Im alkoholischen Destillat bestimmt. 